\title{
Coarse-grained models for frontal photopolymerization with evolving conversion profile
}

\author{
Aloisius R. Purnama, Matthew G. Hennessy, Alessandra Vitale, and João T. Cabral* \\ Department of Chemical Engineering, Imperial College London, \\ London SW7 2AZ, United Kingdom
}

November 5, 2018

\begin{abstract}
We introduce a series of 'minimal' models to describe a common light-driven directional solidification process, known as frontal photopolymerization (FPP), focusing on experimental observables: the solidification kinetics, light attenuation and spatio-temporal monomer-to-polymer conversion. Specifically, we focus on FPP propagation that yields conversion profiles that are not invariant with time, and which cannot be simply described by the presence of mass or heat diffusion. The models are assessed against experimental data for the photopolymerization of a model trimethacrylate system. We find that the simplest model, comprising a single equation of motion for the conversion fraction $\phi$ and a generalized Beer-Lambert law, can only describe the experimental data by assuming an unphysical variation in optical absorption. Introducing a $\phi$-dependent reaction constant $K_{\text {eff }}$ is found to require a time dependence, regardless of the functional form in $\phi$. We conclude by introducing a 'minimal' chemical model, which is based on a simple three-step reaction scheme involving the spatio-temporal evolution of the photoinitiator fraction, relative fraction of radicals, and monomer conversion fraction, that is able to capture the experimental data with a small number of parameters and under reasonable FPP assumptions. Our framework provides important predictive ability for ubiquitous solidification and patterning processes, including 3D printing, via photopolymerization.
\end{abstract}

${ }^{*}$ Email: j.cabral@ic.ac.uk 


\section{Introduction}

Photopolymerization is a powerful and versatile directed solidification process, ${ }^{[1-4]}$ employed in fields ranging from coatings ${ }^{[5,6]}$ and electronics, to dentistry ${ }^{[7]}$ and $3 \mathrm{D}$ printing ${ }^{[8,9]}$. In particular, the use of photopolymerization in 3D printing is growing rapidly due to high speed and precision of stereolithographic (SLA), digital light processing (DLP) ${ }^{[6]}$ and emerging continuous patterning processes ${ }^{[10]}$.

While a monomer-to-polymer conversion profile is generally imperceptible in thin film lithography, curing gradients appear when polymerizing thick monomer baths (e.g. in 3D printing via photopolymerization). In particular, under conditions of strong light attenuation and limited mass and thermal diffusion, a sharp monomer-to-polymer conversion profile develops and propagates as a planar front normal to the illuminated surface. This light-driven process is referred to as frontal photopolymerization (FPP), and shares a number of features with auto-catalytic frontal polymerization ${ }^{[11]}$. However, the FPP process can generally be started and stopped by switching on or off the light source and thus, from a manufacturing perspective, FPP offers an attractive and precise means of thickness control in both unbounded and 3D photopolymerization.

The effective exploitation of photopolymerization in manufacturing requires a predictive patterning model capable of computing not only the solidified thickness, but also the conversion profile along the direction of illumination, which in turn affects a range of physical-chemical properties, including density (possible shrinkage), refractive index, and mechanical properties.

Several reactions (photolysis, initiation, propagation, chain transfer, termination) and species (radicals, distribution of molecular masses and branching), as well as possible thermal and mass diffusion processes, and light attenuation contribute to the remarkable spatio-temporal complexity of FPP. Explicit chemical models generally involve a large number of parameters ${ }^{[12,13]}$, often conspicuously difficult to measure experimentally. For instance, the comprehensive model of Goodner and Bowman ${ }^{[13]}$ considers seven reaction steps, mass diffusion of initiator, monomer and inhibitor, thermal diffusion, as well as free volume changes, requiring a total of 45 model parameters. Further, the model complexity and coupling of various proceses limits the physical insight into the dominant mechanisms and thus the practical utilization of such approaches in predictive photo-patterning. A number of of simplified modelling approaches have thus been proposed ${ }^{[13-17]}$, accounting for a subset of reaction steps, polydispersity and diffusive processes with various degrees of complexity. Such simplified models generally enable the elucidation of causal relations between key model parameters, processes and spatio-temporal photoconversion, including solidification kinetics, conversion profile or light attenuation.

With these ideas in mind, we have previously introduced a coarse-grained, minimal, model for FPP ${ }^{[18-20]}$, coupling a simple first order equation of motion for conversion and a generalized BeerLambert law. The model, summarized below, is focused on two key experimental observables, namely 


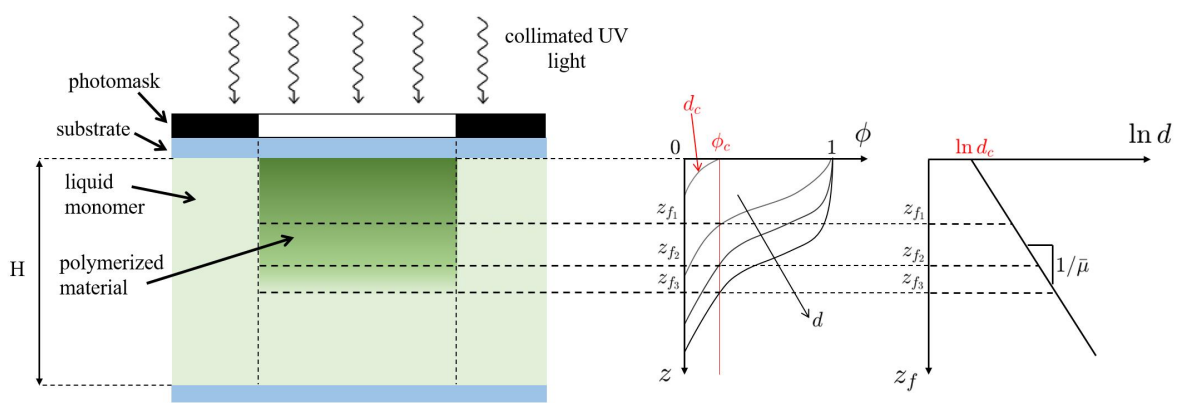

Figure 1: Schematic of the FPP experimental setup showing the collimated UV light, photomask, transparent (glass) substrates, liquid reactive monomer, and polymerized solid material. The dashed lines on the polymer network depict the movement of the photopolymerization front upon increasing irradiation time, $t$, or dose, defined as $d \equiv I_{0} t$, where $I_{0}$ is the light intensity at the illuminated surface. The monomer-to-polymer conversion $\phi$ develops a sigmoidal profile which travels along the $z$-direction as $d$ increases. The front position (or cured thickness) $z_{f}$ corresponds to $\phi(z, t)$ attaining $\phi_{c}$, a critical conversion threshold ('gel point') and generally increases with $\ln d$, after an induction period $d>d_{c}$, with a (logarithmic) gradient inversely proportional to the optical attenuation of the material $\bar{\mu}$.

the patterned (solidified) thickness and the light transmission, and is able to capture not only the front kinetics, but also the conversion profile normal to the illuminated surface for a wide range of systems. A detailled comparison of the main features and differences between the comprehensive and reduced models directly relevant to our work is presented further below, in the context of the new experimental evidence considered.

This approach has been validated with commercial and custom formulated thiol-ene resists, as well as filled systems (with nanoparticles, nanotubes and dyes), and for a wide range of temperature and light intensity ${ }^{[18,19,21,22]}$. However, we have recently found out that certain acrylate systems are not fully described by the model ${ }^{[22]}$. Oxygen inhibition ${ }^{[23]}$ is unlikely to play a role in this process since the monomer bath is confined by impermeable (glass) substrates. While the front position was well captured by our FPP model, exhibiting characteristic logarithmic kinetics with time (or dose), whose slope is set by the optical absorption coefficient $\bar{\mu}$, the spatio-temporal conversion profile deviates from our 'minimal' predictions. Specifically, the experimentally measured fronts were no longer found to be shape-invariant, as predicted by our model. The inclusion of mass diffusion ${ }^{[24]}$ and thermal effects ${ }^{[25]}$ could not satisfactorily account for the spatio-temporal evolution of the conversion data. Despite these differences, we were able to establish a unified growth law, applicable to all systems investigated so far, based on the optical properties and induction time for solidification ${ }^{[22]}$.

The purpose of this paper is to extend our 'minimal' model, comprising a single conversion rate equation (and rate constant), and systematically introduce the simplest modifications able to capture the additional complexities manifested by selected photoactive systems. These include the development 
of travelling fronts whose profile is not shape-invariant, and cannot be accounted by mass or thermal diffusion, as found in a series of acrylates of various functionalities ${ }^{[22]}$. Instead of reducing a comprehensive model ${ }^{[12,13]}$, we build from the simplest 'minimal' FPP model to enable direct elucidation of the physical origin of the observed profile deviations from existing predictions. We seek to simultaneously capture the evolution of the front profile and light attenuation during frontal photopolymerization, while predicting the solidification kinetics relevant for advanced manufacturing.

\section{Experimental}

The reactive mixture is formed by trimethylolpropane trimethacrylate (Sigma-Aldrich) monomer and 1 wt\% photoinitiator 2-hydroxy-2-methyl-1-phenyl-propan-1-one (Sigma-Aldrich). Photocuring was carried out at room temperature by means of a fiber optic UV lamp (Omnicure S1500, equipped with a $365 \mathrm{~nm}$ filter) with an incident light intensity of $3.1 \mathrm{~W} \mathrm{~m}^{-2}$. UV irradiation was performed for different exposure times $t$, so that a wide UV dose window covering $0-5500 \mathrm{~J} \mathrm{~m}^{-2}$ was investigated. Development of the solidified polymer was performed with appropriate selective solvents, acetone, ethanol, or isopropanol, to remove uncrosslinked material. The monomer-to-polymer conversion $\phi$ was measured by FT-IR spectroscopy (Bruker Tensor 27 spectrometer coupled to a Hyperion microscope) by monitoring the decrease of the absorption band of $\mathrm{C}=\mathrm{C}$ (centered at $1640 \mathrm{~cm}^{-1}$ ) reactive functionality along the direction of illumination. The thickness of the crosslinked samples was measured with a reflection optical microscope (Olympus BX41M) or a Dektak-XT stylus profiler (Bruker) and, for large thicknesses, with a digital caliper. The optical attenuation coefficient $\bar{\mu}$ was experimentally measured by recording the evolution of the light transmittance throughout samples of overall constant thickness during the photochemical reaction.

\section{Summary of the 'minimal' FPP model}

We first provide a brief overview of the coarse-grained model introduced by Cabral et al. ${ }^{[18-20]}$ to describe frontal photopolymerization, focusing on experimental observables, namely the patterned height and light transmission. The former is readily obtained by selective dissolution of the liquid monomer-rich phase following illumination (i.e. following 'development'), and measuring the cured thickness of the resulting polymer network. The latter can be easily recorded with a radiometer. A schematic of the FPP process is depicted in figure 1. The monomer-to-polymer conversion $\phi(z, t)$ is the (implicit) order parameter of the model, describing the spatio-temporal conversion of the network, where $\phi=0$ and $\phi=1$ correspond to unpolymerized (i.e., unreacted monomer) and fully polymerized network, respectively, $z$ is the direction normal to the illuminated surface located at $z=0$ (see figure 1 ), and $t$ is the illumination time. The 
rate of conversion is given by

$$
\frac{\partial \phi(z, t)}{\partial t}=K_{\mathrm{eff}}[1-\phi(z, t)] I(z, t)
$$

where $K_{\text {eff }}$ is an effective rate constant of the polymer conversion and $I(z, t)$ is the light intensity through the material, modeled by the Beer-Lambert law:

$$
\frac{\partial I(z, t)}{\partial z}=-\bar{\mu} I(z, t)
$$

where $\bar{\mu}$ is the optical attenuation of the material. Under certain conditions, the monomer and fully converted polymer network exhibit different optical attenuation (e.g., photobleaching). The attenuation is thus generalized to the weighted average of optical attenuation of the unpolymerized, $\mu_{0}$, and fully polymerized material, $\mu_{\infty}$, as

$$
\bar{\mu}=\mu_{0}(1-\phi(z, t))+\mu_{\infty} \phi(z, t)
$$

When the optical attenuation can be assumed to be constant during polymerization, known as photoinvariant polymerization, $\mu_{0}=\mu_{\infty}=\bar{\mu}$, the usual Beer-Lambert law is recovered:

$$
I=I_{0} \exp (-\bar{\mu} z)
$$

where $I_{0}$ is the light intensity at the illuminated surface $(z=0)$. This condition yields an analytical solution for the conversion $\phi$ profile:

$$
\phi(z, t)=1-\exp \left[-K_{\mathrm{eff}} I_{0} t \exp (-\bar{\mu} z)\right]
$$

which has been validated experimentally for a range of thiol-ene systems ${ }^{[21]}$.

Solidification occurs when the conversion $\phi$ exceeds a critical value $\phi_{c}$. The kinetics of the solidification front, located at $z=z_{f}(t)$, can be obtained by solving the implicit equation $\phi\left(z_{f}(t), t\right)=\phi_{c}$, yielding the dependence of the cured thickness $z_{f}$ with irradiation time (or dose). This condition corresponds to the intersection of the $\phi\left(z_{f}, t\right)$ profile, given by eq. 5 , with the constant critical threshold $\phi_{c}$, yielding

$$
z_{f}=\frac{1}{\bar{\mu}} \ln d+\frac{1}{\bar{\mu}} \ln \left[\frac{K_{\mathrm{eff}}}{\ln \left(1 /\left(1-\phi_{c}\right)\right)}\right],
$$

where, $d \equiv I_{0} t$ is the UV dose, shown to be the relevant control parameter ${ }^{[18,19]}$ and enabling the description of experiments with different $I_{0}$. An induction time, or dose $d_{c}$, is required for the conversion to first reach $\phi_{c}$, above which the polymer network forms, and the solid starts propagating from the illuminated surface. This critical dose corresponds to the intersection of $z_{f}(d)$ with $z_{f}=0$, yielding

$$
d_{c}=\frac{\ln \left(1 /\left(1-\phi_{c}\right)\right)}{K_{\mathrm{eff}}}
$$

Despite its simplicity, this minimal framework accurately describes a range of thiol-ene systems ${ }^{[18,22]}$ and mixtures ${ }^{[19]}$. Extensions of the model are able to account for monomer diffusion ${ }^{[24]}$ and thermal effects $^{[25]}$, including heat generation and diffusion, when significant. 


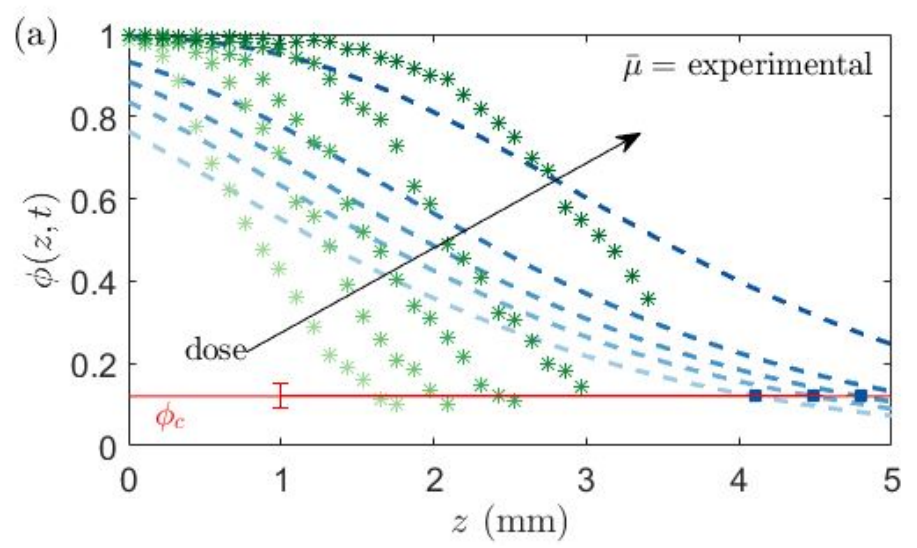

These models are, however, unable to describe certain frontal photopolymerization reactions, for instance, that of a common UV curable multifunctional acrylate system, trimethylolpropane trimethacrylate added with the photoinitiator 2-hydroxy-2-methyl-1-phenyl-propan-1-one, considered here, which we refer to as 'trimethacrylate' throughout the remainder of the paper. For illustration, we plot in figure 2a the experimentally measured $\phi(z, t)$ by cross-sectional FT-IR microscopy, as described previously ${ }^{[22]}$, with the best fit to eq. 5 , imposing the $\bar{\mu}$ obtained experimentally. Evidently, with only $K_{\text {eff }}$ as the fitting parameter, the minimal FPP model is unable to describe the conversion data, which have a significantly sharper profile. Reasonable data fits can be obtained by assuming a large temporal variation in $\bar{\mu}^{[22]}$; however, this is incompatible with experimental data and thus only useful for descriptive purposes. We note, however, that a correct relationship between $z_{f}$ and dose can be obtained by imposing the measured $d_{c}$ (which depends on $K_{\text {eff }}$ ) and the experimental $\bar{\mu}$, which sets the logarithmic slope of the solidification kinetics. This is illustrated in figure $2 \mathrm{~b}$, where the blue squares and dashed line correspond to the model prediction (using the 'minimal' FPP model) with the measured $\bar{\mu}$, and the green points and solid line correspond to the experimentally observed kinetics and fit. Figure 2c plots the experimental attenuation coefficients $\bar{\mu}$ as well as those required to yield reasonable fits to the data shown in figure 2a, which are clearly distinct but appear to converge at long times.

This shortcoming of the 'minimal' models discussed above is expected, given that we employ a single, first-order rate equation (eq. 1) to describe a complex multi-step process, from photolysis to termination, that includes both polymerization and crosslinking. Multifunctional systems generally exhibit two reaction regimes: an autoacceleration at lower $\phi$, followed by an autodeceleration towards reaction completion ${ }^{[26]}$. Thus, we next consider the simplest extension of the model with a rate constant that depends on conversion $\phi$. This seems plausible particularly in the context of the TrommsdorffNorrish effect, the autoacceleration of a free radical polymerization reaction (i.e., the polymerization rate increases despite the monomer consumption), due to the reduction in radical mobility, as a result of the progression of polymerization and crosslinking, and thus the slowing down of termination ${ }^{[1,2,11]}$. 

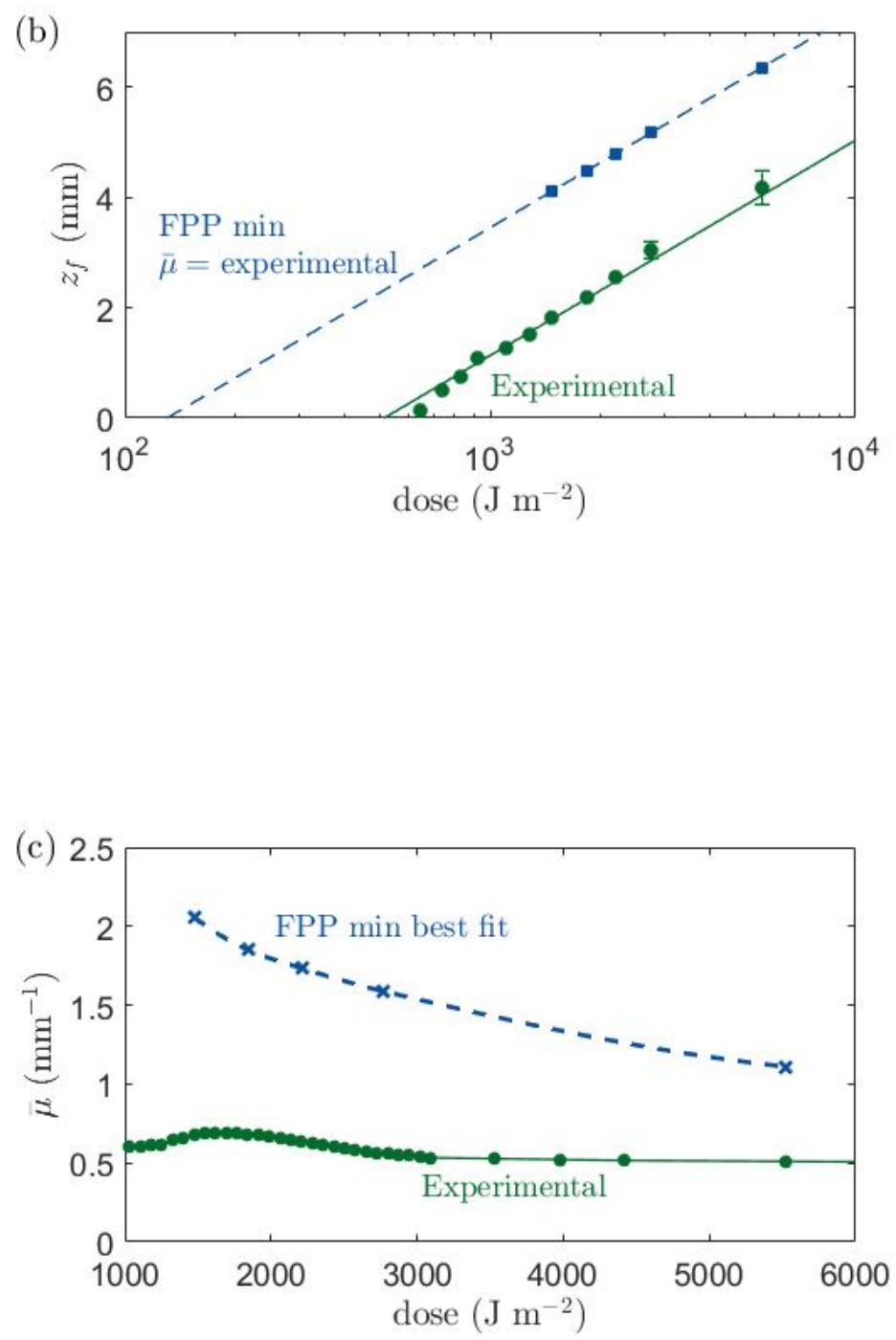

Figure 2: Comparison between the photopolymerization of a trimethacrylate system and the minimal FPP model (FPP min). (a) Conversion profile $\phi(z, t)$ experimental data (symbols) and model prediction (dashed lines), using the experimentally measured absorption coefficient $\bar{\mu}=0.59 \mathrm{~mm}^{-1}$ at the dose range of 1473.6$5526.0 \mathrm{~J} \mathrm{~m}^{-2}$. The horizontal red line corresponds to $\phi_{c}$, the solidification threshold ('gel point') and the typical error bar is included. Blue squares correspond to the intersection between the modeled $\phi(z, t)$ and $\phi_{c}$, yielding the solidified thickness $z=z_{f}$, clearly exceeding the measured thickness. (b) Front position $z_{f}$ dependence on $\ln d$ for both experimental data (green dots and solid line) and model prediction (blue squares and dashed line) obtained from (a). (c) Experimentally measured $\bar{\mu}$ as a function of dose. For illustration, the $\mu$ parameters required to yield satisfactory fits to the experimental data are also included, albeit clearly incompatible with the measurements. 


\section{An FPP model with a $\phi$-dependent $K_{\text {eff }}$}

Inspired by the experimental data, we tentatively introduce a rate constant with a dependence on $\phi$, seeking to account for two possible mechanisms, whereby one dominates as the polymerization starts (low $\phi$ ) and the other towards completion (high $\phi$ ). Thus, we introduce an effective rate constant, $K_{\text {eff }}$, which can be expressed as the weighted average of the rate constant at low $\phi, K_{0}$, and high $\phi, K_{1}$ :

$$
K_{\mathrm{eff}}=K_{0}(1-\phi)+K_{1} \phi
$$

Upon substitution of eq. 4 and 8 into eq. 1, we obtain a rate equation, which we term the 'FPP $K(\phi)$ model':

$$
\frac{\partial \phi}{\partial t}=\left[K_{0}(1-\phi)+K_{1} \phi\right](1-\phi) I_{0} \exp (-\bar{\mu} z)
$$

In our formalism, the actual 'reaction rate' is given by $K_{\text {eff }}(1-\phi)$ and, as expected, vanishes upon completion $(\phi=1)$. Thus, the precise functional form of $K_{\text {eff }}$ is only expected to influence the reaction rate at lower values of $\phi$.

Equation 8 is analogous to eq. 3 for optical absorption and implicitly suggests a two-step FPP model, albeit still described with a single conversion order parameter $\phi$. The model is solved numerically with two fitting parameters, $K_{0}$ and $K_{1}$, yielding good agreement with both the experimental conversion $\phi(z, t)$ and front position $z_{f}$ data, as shown in figure $3 \mathrm{a}$ and $\mathrm{b}$. However, this can only be achieved by varying $K_{0}$ and $K_{1}$ with UV dose (or time) as illustrated in figure 3c, which is unsatisfactory.

To gain insight into the physical meaning of the time-dependent rate constants, we take the first pair of $K_{0}$ and $K_{1}$ values given by $3.7 \times 10^{-5}$ and $5.6 \times 10^{-3} \mathrm{~m}^{2} \mathrm{~J}^{-1}$, respectively, and predict the conversion profiles at subsequent doses. Based on these results (not shown), the theoretical conversion profiles are found to be too sharp and they consistently overestimate the experimental conversion fraction. That is, for a given dose, the theoretical conversion profile appears to have propagated further into the bath than what is observed experimentally. Thus, we can interpret the time variation of $K_{0}$ and $K_{1}$ shown in figure $3 \mathrm{c}$ as follows: the late-stage broadening occurs due to a decrease in $K_{1}$ while an increase in $K_{0}$ captures the slowing of front propagation. The time-varying ratio $K_{1} / K_{0}$ of the two rate parameters, which decreases over time is therefore needed for the description of the data.

To further explore the dependence of $K_{\mathrm{eff}}$ on time and conversion fraction, the front profiles $\phi(z, t)$ and $K_{\text {eff }}(z, t)$ are superposed and plotted in figure 4 . The light (yellow) region at high $\phi$ and early times corresponds to narrower conversion profiles and faster propagation, as is required to account for the experimental data.

At all doses, parameter $K_{1}$ is consistently higher than $K_{0}$, compatible with an autoacceleration effect where the rate of free radical termination becomes diffusion limited ${ }^{[4]}$. As $K_{1} \gg K_{0}, K_{\text {eff }}$ in eq. 8 can 

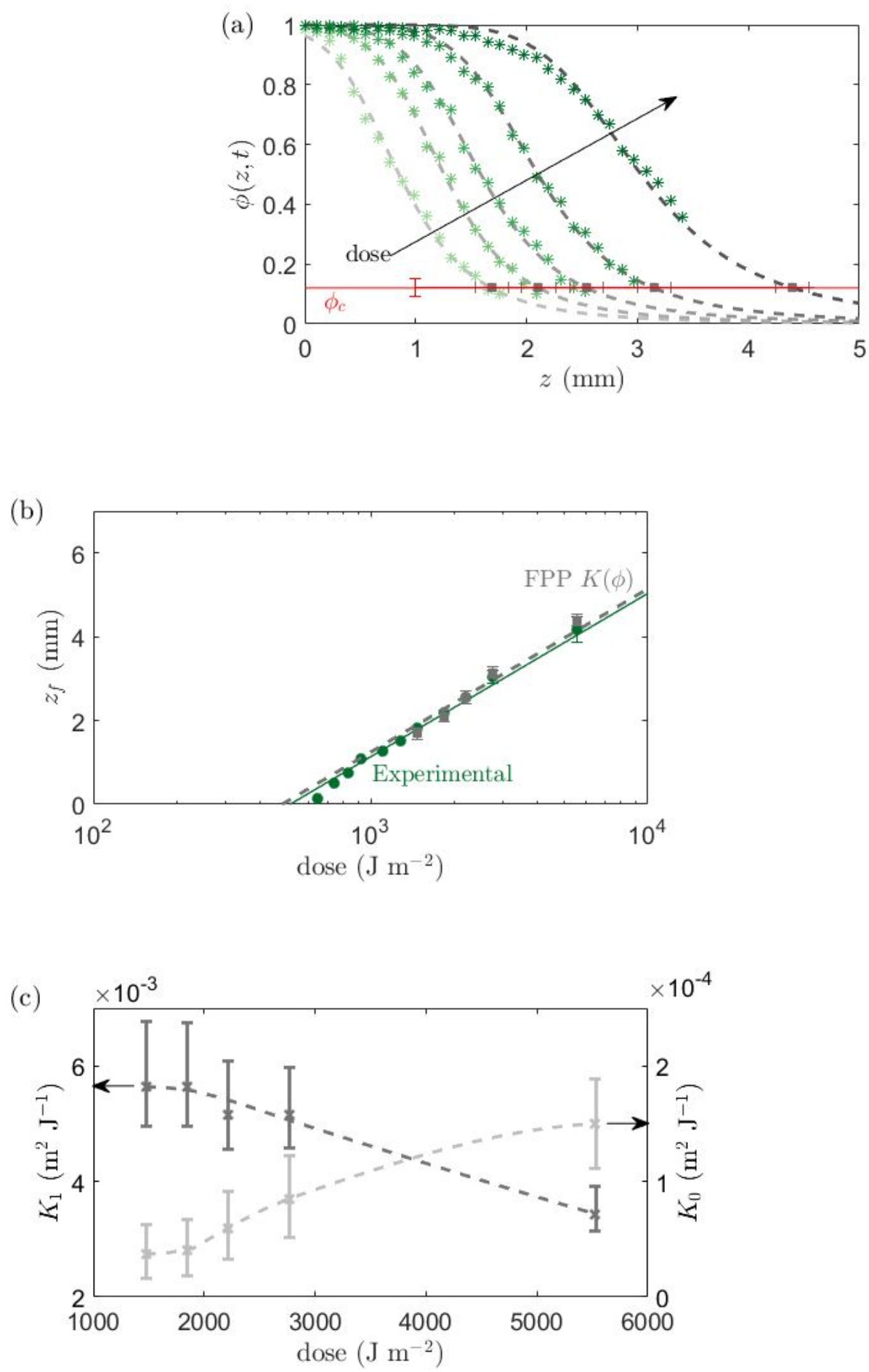

Figure 3: FPP two-step model results (FPP K $(\phi)$, grey dashed lines and points) in good agreement with experimental (a) $\phi(z, t)$ and (b) $z_{f}(t)$ kinetics (green dots and solid line) as a function of dose for the trimethacrylate system. (c) The fitting parameters using the linear functional form whereby both $K_{0}$ and $K_{1}$ are changing with dose (time). The error bars indicate the range of parameters compatible with the front kinetics data within $z_{f} \pm 0.15 \mathrm{~mm}$. 


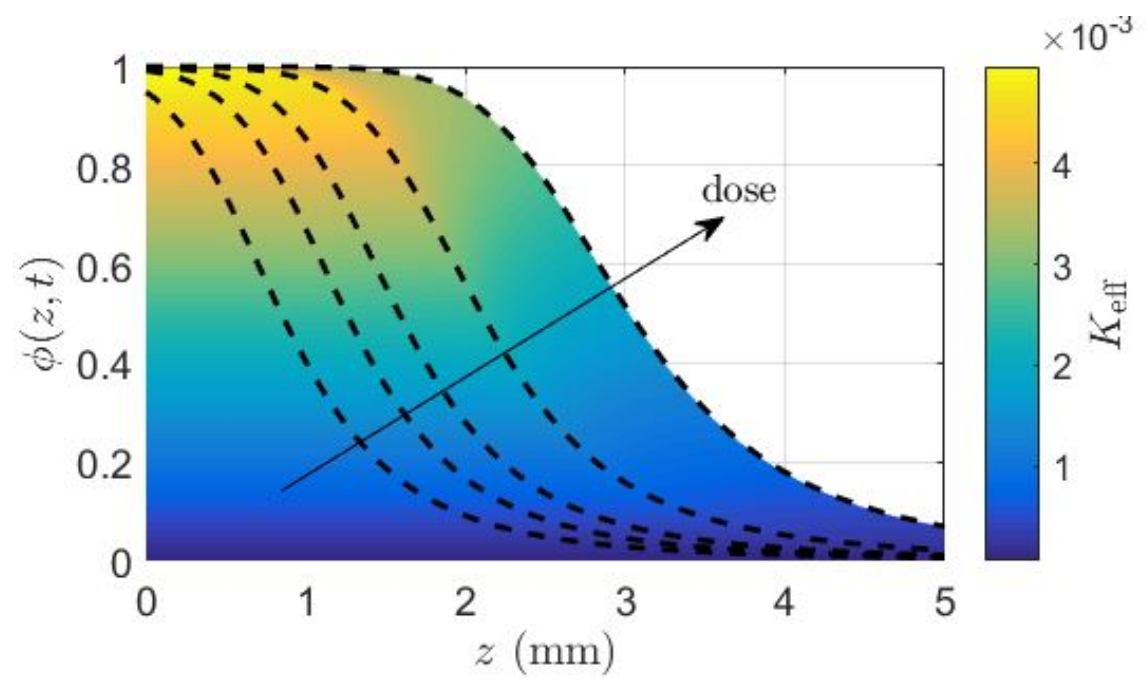

Figure 4: Superposition of front profile $\phi(z, t)$ with the $K_{\text {eff }}$ values required to describe the data using the FPP two-step model (FPP $\mathrm{K}(\phi))$. $K_{\text {eff }}$ is displayed as a color map, with scale reported on the right, indicating that a dependence on both $\phi$ and $t$ is needed.

be written as

$$
K_{\text {eff }}=K_{0}+K_{1} \phi
$$

The linear form of $K_{\text {eff }}$ is chosen for simplicity and effectiveness, but different functional forms, including a power-law or hyperbolic tangent profiles, were also considered, given by

$$
K_{\mathrm{eff}}=K_{0}+K_{1} \phi^{a}
$$

and

$$
K_{\mathrm{eff}}=\frac{K_{1}+K_{0}}{2}+\frac{K_{1}-K_{0}}{2} \tanh \left(\frac{\phi-\phi_{*}}{w}\right),
$$

where the exponent $a$ controls the curvature of $K_{\text {eff }}$, and $\phi_{*}$ and $w$ are the mid-point and width of the sigmoidal $K_{\text {eff }}$ curve, respectively. These functional forms are fitted to the experimental data in figure 5a. Of all of the functional forms for $K_{\text {eff }}$ that we explored, only the linear $(a=1)$ and the hyperbolic tangent (with $\phi_{*}=0.43$ and $w=0.26$ ) were able to yield satisfactory agreement with the data. When using a hyperbolic tangent function, the parameters $K_{0}$ and $K_{1}$ change similarly as when a linear form is used; that is, $K_{0}$ increases while $K_{1}$ decreases with dose. In figure 5 b, the $K_{\text {eff }}$ of each functional form is plotted as a function of $\phi$ and the maximum $K_{\text {eff }}$ value is normalized to 1 because each functional form yields a different set of $K_{0}$ and $K_{1}$.

We conclude that no equation of the form of eq. 1, regardless of the $\phi$-dependence of the effective rate constant $K_{\text {eff }}$, can reproduce the experimental data. Moreover, further extensions to the minimal model in eq. 1 , which multiply the effective rate constant $K_{\text {eff }}(\phi)$ by a dimensionless decreasing function $f(t)$ 

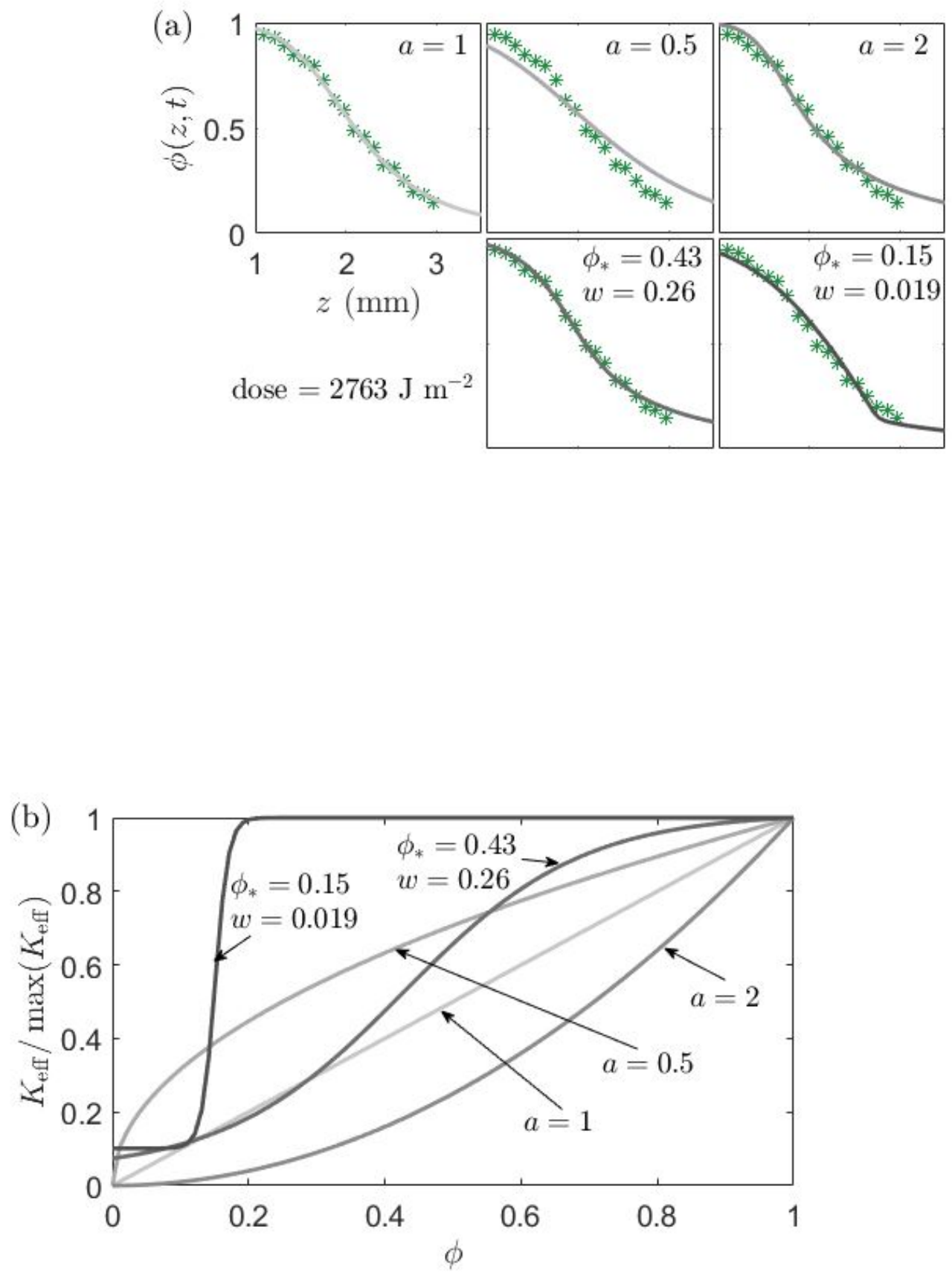

Figure 5: (a) Evaluation of different functional forms, given by eq. 11 and 12 to a representative conversion profile. The linear form $(a=1)$ is the simplest function and also that which produces the closest agreement with data. (b) The normalized $K_{\text {eff }} / \max \left(K_{\text {eff }}\right)$ dependence on $\phi$, corresponding to the profiles in (a). 
Scheme 1: The simplified photopolymerization scheme that is used to formulate a 'minimal' FPP chemical model. This scheme consists of three reactions between photoinitiator (PI), unconverted monomer (M), active radical species $\left(R^{\bullet}\right)$, and grouped inactive species $(R)$; see text for further details.

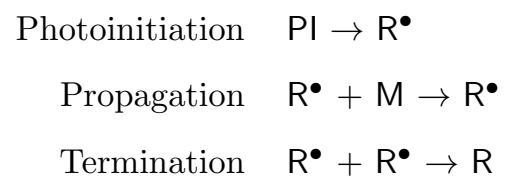

that represents the slowing of conversion, are also unable to provide satisfactory agreement between experiment and theory. This is because models with time-modulated rate constants of the form $K_{\text {eff }}(\phi) f(t)$ lead to time-invariant conversion profiles, in contrast to experimental observations. A mathematical analysis of such models reveals that the functional form of $K_{\mathrm{eff}}(\phi)$ selects the shape of the time-invariant conversion profile while the function $f(t)$ controls the horizontal translation of these profiles and, in particular, the advancement of the solidification front $z_{f}(t)$. In the next section, we introduce an additional equation that can resolve this issue, in a minimal model motivated by the photopolymerization reaction chemistry.

\section{A 'minimal' FPP chemical model}

To simultaneously capture the overall decrease in the rate of conversion and the broadening of the conversion profile, a minimal-chemical model that explicitly incorporates additional species and reaction steps is developed. This model aims to bridge the gap between minimal and comprehensive physicalchemical models of FPP and, indeed, can be systemically derived from the latter under appropriate conditions that will be detailed below.

The minimal-chemical model is based on a simplified photopolymerization scheme, as shown in scheme 1, that involves photoinitiators (PI); unconverted monomer (M); a 'grouped' active radical species, which accounts for primary radicals and polymer chains of varying lengths $\left(R^{\bullet}\right)$, and a 'grouped' inactive species (R). The first step in the photopolymerization scheme, which we refer to as 'photoinitiation', describes the generation of primary radicals via photolysis and the initiation of active polymer chains due to reactions between primary radicals and monomers. In the spirit of developing the simplest 'minimal' FPP chemical model, we assume that the photoinitiator forms just one (instead of two, as usually found in more complex models) reactive species that can then further react. The second reaction in the scheme is a propagation-like step that describes the conversion of monomer into polymer chains of increasing length. It is important to note that, due to the multifunctional nature of the analyzed system, crosslinked chains are formed during this step. Lastly, the termination step captures the reaction between two active polymer chains, yielding a longer, albeit inactive, chain. 
The mathematical formulation of the minimal-chemical model consists of equations which govern the spatio-temporal evolution of the photoinitiator fraction, $p=[\mathrm{PI}] /[\mathrm{PI}]_{0}$, relative fraction of radicals, $r=\left[\mathrm{R}^{\bullet}\right] /[\mathrm{PI}]_{0}$, and monomer conversion fraction, $\phi=1-[\mathrm{M}] /[\mathrm{M}]_{0}$, where $[\mathrm{PI}]_{0}$ and $[\mathrm{M}]_{0}$ denote the initial concentrations of photoinitiator and monomer, respectively. The rate of photoinitiation, $R_{i}$, is assumed to be proportional to the local photoinitiator fraction and light intensity, giving $R_{i}=K_{i} p I$, where $K_{i}$ is a coarse-grained rate constant. The rate of propagation, $R_{p}$, employs the same conversion-dependent rate coefficient $K_{\text {eff }}(\phi)$ introduced previously, given by eq. 8 , and thus can be written as $R_{p}=K_{\text {eff }}(\phi)(1-\phi) r$. Finally, the rate of termination, $R_{t}$, is taken to be $R_{t}=K_{t} r^{2}$.

To further simplify the model, the fraction of photoinitiator $p$ is assumed to be spatially uniform, which can be made rigorous in the limit where the rate of mass diffusion is large compared to the rate of front propagation ${ }^{[24]}$. Under these conditions, it can be shown that $p$ must decay exponentially in time according to

$$
p(t)=\exp \left(-K_{i} \bar{I} t\right)
$$

where $\bar{I}$ is the depth-averaged light intensity given by

$$
\bar{I}=\frac{1}{H} \int_{0}^{H} I(z) \mathrm{d} z=\frac{I_{0}}{\bar{\mu} H}[1-\exp (-\bar{\mu} H)],
$$

where, $H$ is the height of the monomer bath shown in figure 1. Although photoinitiators are consumed, we treat the intensity as being constant in time with a time-independent absorption coefficient $\bar{\mu}$, consistent with experimental observations, as shown in figure 2c. Such approximation is expected to be valid for photoresists comprising weakly absorbing photoinitiators.

The equations of motion for the fraction of radicals $r$ and converted monomer $\phi$ are given by

$$
\begin{aligned}
& \frac{\partial r}{\partial t}=K_{i} p(t) I_{0} \exp (-\bar{\mu} z)-K_{t} r^{2}, \\
& \frac{\partial \phi}{\partial t}=K_{\mathrm{eff}}(\phi)(1-\phi) r,
\end{aligned}
$$

which have the initial conditions $\phi(z, 0)=r(z, 0)=0$. A comparison of eq. 15b with the minimal model eq. 1 reveals that the radical fraction $r$ now plays the role of light intensity in monomer conversion. As the sharpness of the conversion profile in the minimal model is set by the gradient in light intensity ${ }^{[24]}$, as measured through the absorption coefficient $\bar{\mu}=-\mathrm{d}\left[\ln \left(I / I_{0}\right)\right] / \mathrm{d} z$, we can expect that the gradient of the radical fraction, $\nu \equiv-\mathrm{d}(\ln r) / \mathrm{d} z$, will thus control the shape of the conversion profile in the minimal-chemical model.

The influence of photoinitiation and termination on the conversion process can be understood by first considering the limit of negligible termination, $K_{t} r^{2} \ll K_{i} I$. In this case, the fraction of radicals increases exponentially in time,

$$
r(z, t)=\left(I_{0} / \bar{I}\right)\left[1-\exp \left(-K_{i} \bar{I} t\right)\right] \exp (-\bar{\mu} z)
$$




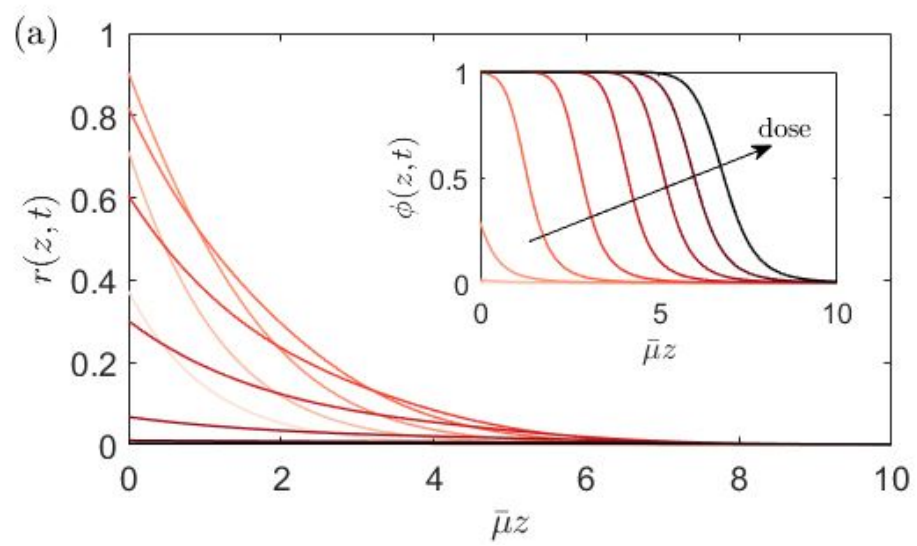

The gradient in radical fraction is independent of time and equal to $\nu=\bar{\mu}$. Therefore, the conversion profiles remain time invariant and their sharpness can be directly controlled via the absorption coefficient $\bar{\mu}$. The time dependence of the radical fraction has important consequences for the propagation of the solidification front, however. In the initial phase of photoinitiation given by $t \ll\left(K_{i} \bar{I}\right)^{-1}$, the fraction of radicals increases linearly with time, $r(z, t) \sim K_{i} I_{0} t \exp (-\bar{\mu} z)$, and we find that the solidification front propagates according to the law $z_{f}(t) \sim(2 / \bar{\mu}) \ln t$. In the late stages of photoinitiation, $t \gg\left(K_{i} \bar{I}\right)^{-1}$, the fraction of radicals reaches a steady state due to the depletion of photoinitiator,

$$
r(z, t) \sim\left(I_{0} / \bar{I}\right) \exp (-\bar{\mu} z),
$$

and the front propagates according to the previously established law of $z_{f}(t) \sim(1 / \bar{\mu}) \ln t$. Although the initial propagation of the front appears to occur twice as rapidly as compared to the long-term rate, there is a considerable increase in the induction time due to the need to generate radicals before conversion can take place, which slows the overall rate of front propagation.

If the photoinitiation stage is fast relative to propagation, then the fraction of radicals quickly settles into its steady-state profile given by eq. 17 before there is significant conversion of monomer. In this case, eq. 17 can be directly inserted into eq. 15b, which recovers the FPP minimal and $K(\phi)$ models of the previous sections. Thus, the previous models are valid in the limit of negligible termination and fast generation of radicals.

When termination is non-negligible and occurs at a rate that is commensurate with photoinitiation, then, initially, the radical fraction increases according to eq. 16 and hence yields a profile that exponentially decreases with distance from the illuminated surface. However, termination will start to counteract radical generation at a rate that increases with the local radical fraction, leading to flattening of the exponential profile (figure 6a) and a broadening of the conversion fraction (figure 6b). For low values of $\phi_{c}$, the critical dose is exceeded before termination begins to decrease the radical fraction. Therefore, the front propagation is not significantly affected by termination and it exhibits the same two time regimes that were observed when termination is negligible (figure 6c). For larger values of $\phi_{c}$ or the rate of 

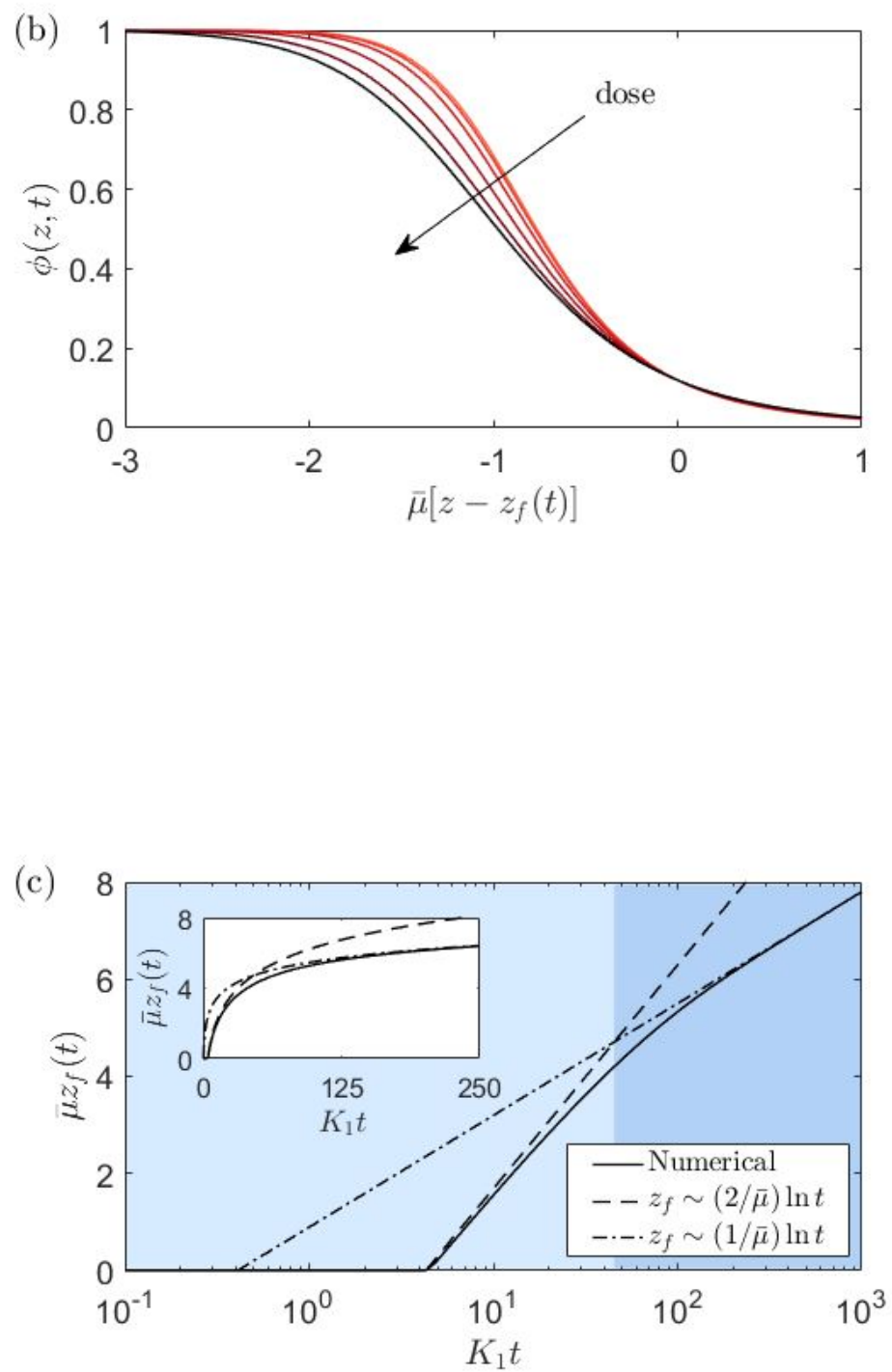

Figure 6: Numerical simulation of the minimal-chemical model. (a) The evolution of the radical (main panel) and conversion fraction (inset) with dose. (b) Conversion profile as viewed from a frame of reference that travels with the solidification front $z_{f}(t)$. The conversion profile broadens as the gradient in the radical fraction decreases. (c) The propagation of the solidification front can be split into two regimes. In the first regime (lighter shading), the radical fraction is increasing with time, leading to the front propagating according to the law $z_{f}(t) \sim(2 / \bar{\mu}) \ln t$. In the second regime (darker shading), the radical fraction has reached a steady state and the time dependence of the solidification front is given by $z_{f}(t) \sim(1 / \bar{\mu}) \ln t$. The transition between the two regimes occurs when $t \sim\left(K_{i} \bar{I}\right)^{-1}$. Parameter values used in the simulation are $K_{i} I_{0} / K_{1}=K_{t} / K_{1}=0.4, K_{0} / K_{1}=0.01, \bar{\mu} H=11.8$, and $\phi_{c}=0.12$. 


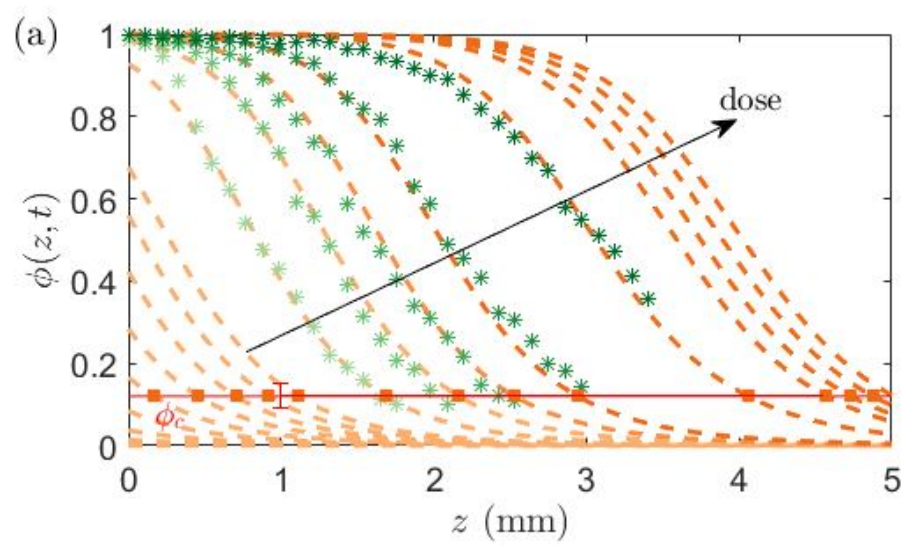

termination $K_{t}$, the conversion process is more strongly affected by termination and, as a result, the relationship between $z_{f}$ and time (or dose) becomes non-logarithmic.

The minimal-chemical model has four fitting parameters, namely $K_{0}, K_{1}, K_{i}$, and $K_{t}$, and fits the experimental data remarkably well for both the spatio-temporal conversion $\phi(z, t)$ and front kinetics $z_{f}(t)$, shown in figure 7 . As expected, $K_{1} \gg K_{0}$, which is required for the model to capture the sharpness of the initial conversion profiles. Furthermore, the late-stage broadening of the conversion profile, which is due to the flattening of radical fraction caused by termination, is well described by the minimal-chemical model. From figure $7 \mathrm{~b}$, it can be seen that all but the first three data points for $z_{f}$ lie closely on the $z_{f}(t) \sim(1 / \bar{\mu}) \ln t$ curve, suggesting that the predicted transition from $z_{f}(t) \sim(2 / \bar{\mu}) \ln t$ growth rate occurs very early in the solidification process. For this to be the case, the consumption of photoinitiators and generation of primary radicals must be fast compared to monomer conversion in this trimethacrylate system. Indeed, using the parameter values from the fit, namely $K_{i}=4.5 \times 10^{-2} \mathrm{~m}^{2}$ $\mathrm{J}^{-1}$ and $K_{1}=7.2 \times 10^{-3} \mathrm{~s}^{-1}$, and the experimentally measured incident intensity $I_{0}=3.1 \mathrm{~W} \mathrm{~m}^{-2}$, we find that $K_{i} I_{0} / K_{1} \sim 20$, implying that the free radical generation is roughly 20 times faster than maximal rate of conversion. This is consistent with the high concentration of photoinitiator used and the relatively low reactivity of methacrylic groups. Similarly, the small relative time scale of conversion to termination, as measured by the ratio $K_{t} / K_{1} \sim 0.2$, is representative of a system whereby the broadening of the conversion profile occurs after it fully develops and begins to propagate into the monomer-rich bath.

\section{Comparison with existing models}

The literature contains a number of theoretical models of photopolymerization that range in their complexity. Terrones and Pearlstein ${ }^{[17]}$ developed a model for the photoinitiation reaction of scheme 1, which focuses on the spatiotemporal evolution of the photoinitiator concentration. Their model is based 


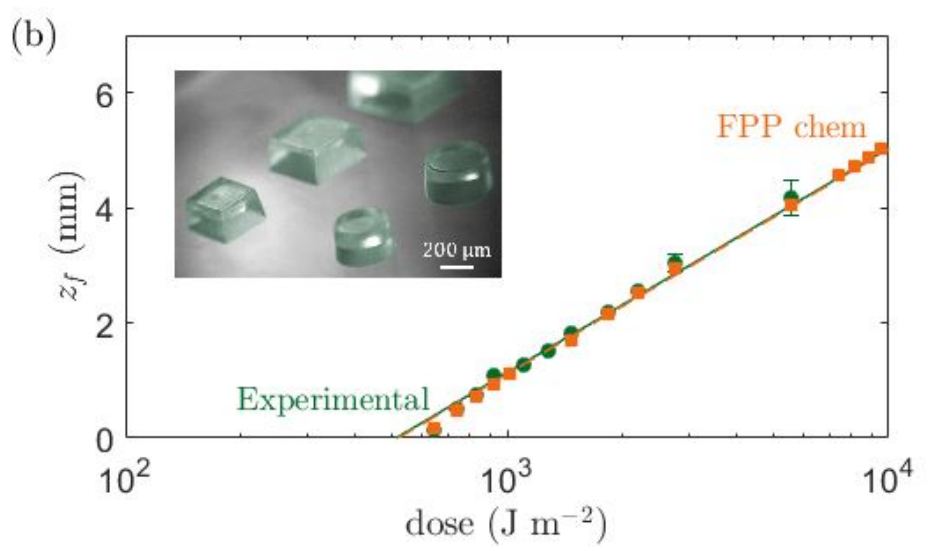

Figure 7: (a) Fits of the minimal-chemical model (FPP chem) to experimental data for the conversion fraction with predicted profiles at earlier and later doses, and (b) the front position, corresponding to fitted parameters $K_{0}=4.2 \times 10^{-6} \mathrm{~s}^{-1}, K_{1}=7.2 \times 10^{-3} \mathrm{~s}^{-1}, K_{i}=4.5 \times 10^{-2} \mathrm{~m}^{2} \mathrm{~J}^{-1}$, and $K_{t}=1.7 \times 10^{-3} \mathrm{~s}^{-1}$. The predictive capability of the minimal-chemical model facilitates the controlled patterning of three-dimensional structures (inset), whose footprint and height can be controlled via the photomask geometry and irradiation time, respectively.

on a photobleaching system and does not consider diffusion of the photoinitiating species. Photobleaching systems are expected to have an optical attenuation coefficient that decreases with time, in contrast to what is observed in the acrylate systems considered here; see figure 2 (c). Due to the absence of mass diffusion in their model, the concentration of photoinitiator can settle into a travelling-wave profile, which is markedly different from the uniform profiles that are considered here. Terrones and Pearlstein ${ }^{[16]}$ have also presented an extended model that captures the propagation and termination reactions. More specifically, their extended model considers an arbitrary number of propagation reactions of the form $\mathrm{R}_{m}^{\bullet}+\mathrm{M} \rightarrow \mathrm{R}_{m+1}^{\bullet}$, with $m \geq 0$, where $\mathrm{R}_{m}^{\bullet}$ corresponds to an active radical species with chain length $m$ and mass fraction $r_{m}$. However, the rate equations for these propagation reactions are summed to yield a 'net' rate equation that is mathematically identical to equation (15a). Importantly, this 'net' rate equation enables the radical fraction $r$ in equation (15a) to be precisely defined as the sum of active radical mass fractions, that is,

$$
r(z, t) \equiv \sum_{m=0}^{\infty} r_{m}(z, t) .
$$

A key distinction between the extended model of Terrones and Pearlstein ${ }^{[16]}$ and the minimal-chemical model considered here (eqns. (13)-(15)) is that the former assumes the propagation reactions are in a steady state and there is no, or relatively slow, diffusion of photoinitiator. The minimal-chemical model, however, is based on the opposite limit whereby diffusion of photoinitiator is fast relative to the propagation reaction so that the steady-state approximation does not hold. Had we also neglected diffusion of photoinitiator and invoked the steady-state approximation, then an analytical solution to 
the governing equations can be found, although this is not able to accurately capture the experimental conversion profiles nor the propagation of the polymerization front $z_{f}$. The discrepancies between theory and experiment can be explained by the fact that in the steady-state limit, the rate of monomer conversion, i.e., the right-hand side of eqn. (15b), becomes proportional to $I^{1 / 2}$, effectively halving the value of the optical attenuation coefficient. Consequently, the sharpness of the theoretical conversion profiles is reduced by a factor of two and the rate of front propagation is doubled.

The minimal-chemical model (13)-(15) and the models of Terrones and Pearlstein ${ }^{[16,17]}$ are simplifications of the comprehensive physical-chemical model proposed by Goodner and Bownan ${ }^{[13]}$. The latter considers thermal effects such as exothermic heat release and diffusion, multicomponent mass diffusion, and the temperature and free-volume dependence of the diffusion and rate coefficients. Moreover, it considers additional reaction steps such as chain inhibition, re-initiation of inactive chains, and primary radical termination. The comprehensive nature of this model leads to governing equations of staggering complexity and which contain a large number of physical parameters, thus limiting its practicality and predicting power. However, Goodner and Bowman's model highlights the multiple processes that simultaneously occur during photopolymerization and gives insight into the many ways in which reduced models, such as the ones presented here, can be extended in order to better capture experimental data.

\section{Conclusion}

The previously reported 'minimal' model ${ }^{[18-20]}$ for frontal photopolymerization (FPP) is extended to describe systems whose front profile is no longer time-invariant during propagation and which cannot be reasonably captured by the inclusion of heat or mass diffusion ${ }^{[24,25]}$. Simple modifications of the coarse-grained model are tested with a trimethacrylate system ${ }^{[22]}$ previously found to depart from our predictions, by contrast with thiol-ene, both neat and filled, systems ${ }^{[18,19,21,22]}$.

A coarse-grained model of FPP, in which the effective rate coefficient $K_{\text {eff }}$ increases with the conversion fraction $\phi$ and takes on two extreme values $K_{0}=K_{\text {eff }}(0)$ and $K_{1}=K_{\text {eff }}(1)$, was introduced in order to capture the expected autoacceleration phenomenon. While this FPP $K(\phi)$ model was able to reproduce the correct conversion profiles, breaking the time invariance of front propagation required the rate coefficients $K_{0}$ and $K_{1}$ to be time dependent. Furthermore, it was observed that there is a late-stage slowing of front propagation that could not be accounted for in a model of this type with constant rate coefficients.

The broadening of the conversion profile and reduction in propagation rate can, however, be captured by a minimal-chemical model that is based on a subset of photopolymerization steps and chemical species. A critical assumption is that a rapid mass diffusion acts to keep the composition of a photoinitiator-like species homogeneous. Consumption of this species near the illuminated surface thus leads to a decrease in its bulk concentration, thereby reducing the bulk generation of radical-like species and hence the rate 
of conversion. Furthermore, we find that the fraction of the radical-like species plays the same role as the light intensity in the minimal FPP model; therefore, the evolving gradient of the radical fraction, which is due in part to a termination-like consumption reaction, drives the broadening of the conversion profiles. A comparison of the minimal chemical model with experimental data shows that the two are in excellent agreement.

Predictive FPP patterning of these systems is thus possible, with the minimal-chemical model accurately describing both the front profile and front position (i.e. the cured thickness) at all conditions. This predictive ability is valuable from a range of applications from solidification of protective coatings and electronic laminates to the high resolution 3D printing of polymers by photopolymerization.

\section{Acknowledgements}

We acknowledge the financial support from the Engineering and Physical Sciences Research Council (EPSRC) [grant numbers EP/K503733/1, EP/L020564/1], and the Royal Society (UK). Data is available on request: please contact polymer-microfluidics@imperial.ac.uk.

\section{References}

[1] G. Odian, "Principles of polymer chemistry," Wiley, New York, 1991.

[2] J.-P. Fouassier, Photoinitiation, photopolymerization, and photocuring: fundamentals and applications. Hanser, 1995.

[3] J.-P. Fouassier and J. E. Rabek, Radiation Curing in Polymer Science and Technology. Elsevier Applied Science: London, 1993.

[4] C. Decker, "Photoinitiated crosslinking polymerisation," Progress in polymer science, vol. 21, no. 4, pp. 593$650,1996$.

[5] M. Sangermano, G. Malucelli, E. Amerio, A. Priola, E. Billi, and G. Rizza, "Photopolymerization of epoxy coatings containing silica nanoparticles," Progress in Organic Coatings, vol. 54, no. 2, pp. 134-138, 2005.

[6] R. Liska, M. Schuster, R. Inführ, C. Turecek, C. Fritscher, B. Seidl, V. Schmidt, L. Kuna, A. Haase, F. Varga, et al., "Photopolymers for rapid prototyping," Journal of Coatings Technology and Research, vol. 4, no. 4, pp. 505-510, 2007.

[7] J. W. Stansbury and M. J. Idacavage, "3d printing with polymers: Challenges among expanding options and opportunities," Dental Materials, vol. 32, no. 1, pp. 54-64, 2016.

[8] C. N. LaFratta, J. T. Fourkas, T. Baldacchini, and R. A. Farrer, "Multiphoton fabrication," Angewandte Chemie International Edition, vol. 46, no. 33, pp. 6238-6258, 2007. 
[9] H. Lipson and M. Kurman, Fabricated: The new world of 3D printing. John Wiley \& Sons, 2013.

[10] J. R. Tumbleston, D. Shirvanyants, N. Ermoshkin, R. Janusziewicz, A. R. Johnson, D. Kelly, K. Chen, R. Pinschmidt, J. P. Rolland, A. Ermoshkin, et al., "Continuous liquid interface production of 3d objects," Science, vol. 347, no. 6228, pp. 1349-1352, 2015.

[11] J. Pojman, "4.38 - frontal polymerization," In Polymer Science A: A comprehensive Reference. Editors: Matyjaszewski K. and Möller M, vol. 4, pp. 957-980, 2012.

[12] E. Andrzejewska, "Photopolymerization kinetics of multifunctional monomers," Progress in polymer science, vol. 26 , no. 4 , pp. $605-665,2001$.

[13] M. D. Goodner and C. N. Bowman, "Development of a comprehensive free radical photopolymerization model incorporating heat and mass transfer effects in thick films," Chemical Engineering Science, vol. 57, no. 5, pp. 887-900, 2002.

[14] V. V. Ivanov and C. Decker, "Kinetic study of photoinitiated frontal polymerization," Polymer international, vol. 50, no. 1, pp. 113-118, 2001.

[15] B. L. Rytov, V. B. Ivanov, V. V. Ivanov, and V. M. Anisimov vol. 37, p. 5695, 1996.

[16] G. Terrones and A. J. Pearlstein, "Effects of kinetics and optical attenuation on the completeness, uniformity, and dynamics of monomer conversion in free-radical photopolymerizations," Macromolecules, vol. 34, no. 26, pp. 8894-8906, 2001.

[17] G. Terrones and A. J. Pearlstein, "Effects of optical attenuation and consumption of a photobleaching initiator on local initiation rates in photopolymerizations," Macromolecules, vol. 34, no. 10, pp. 3195-3204, 2001.

[18] J. T. Cabral, S. D. Hudson, C. Harrison, and J. F. Douglas, "Frontal photopolymerization for microfluidic applications," Langmuir, vol. 20, no. 23, pp. 10020-10029, 2004.

[19] J. T. Cabral and J. F. Douglas, "Propagating waves of network formation induced by light," Polymer, vol. 46, no. 12, pp. 4230-4241, 2005.

[20] J. A. Warren, J. T. Cabral, and J. F. Douglas, "Solution of a field theory model of frontal photopolymerization," Physical Review E, vol. 72, no. 2, p. 021801, 2005.

[21] A. Vitale, M. G. Hennessy, O. K. Matar, and J. T. Cabral, "Interfacial profile and propagation of frontal photopolymerization waves," Macromolecules, vol. 48, no. 1, pp. 198-205, 2014.

[22] A. Vitale, M. G. Hennessy, O. K. Matar, and J. T. Cabral, "A unified approach for patterning via frontal photopolymerization," Advanced Materials, vol. 27, no. 40, pp. 6118-6124, 2015.

[23] D. Dendukuri, P. Panda, R. Haghgooie, J. M. Kim, T. A. Hatton, and P. S. Doyle, "Modeling of oxygeninhibited free radical photopolymerization in a pdms microfluidic device," Macromolecules, vol. 41, no. 22, pp. 8547-8556, 2008. 
[24] M. G. Hennessy, A. Vitale, O. K. Matar, and J. T. Cabral, "Controlling frontal photopolymerization with optical attenuation and mass diffusion," Physical Review E, vol. 91, no. 6, p. 062402, 2015.

[25] M. G. Hennessy, A. Vitale, J. T. Cabral, and O. K. Matar, "Role of heat generation and thermal diffusion during frontal photopolymerization," Physical Review E, vol. 92, no. 2, p. 022403, 2015.

[26] C. N. Bowman and C. J. Kloxin, "Toward an enhanced understanding and implementation of photopolymerization reactions," AIChE Journal, vol. 54, no. 11, pp. 2775-2795, 2008. 